\title{
Comparative Analysis of the Symptomatology of Children with Lower Urinary Tract Dysfunction in Relation to Objective Data
}

\author{
Ubirajara Barroso Jr, Thiago Nova, Anderson Dultra, Patricia Lordelo, Juarez Andrade, \\ Antonio J. Vinhaes
}

Section of Urology, Federal University of Bahia, Salvador, Bahia, Brazil

\begin{abstract}
Objectives: To assess the clinical presentation of children with lower urinary tract dysfunction (LUTD) relating to objective examination data.

Materials and Methods: Forty-four children (36 girls and 8 boys with mean age of 6.8 years) with LUTD were prospectively assessed through a specific questionnaire that analyzed clinical presentation of those patients. These data were then compared to objective data, such as micturition diary and uroflowmetry with electromyography.

Results: A urinary tract infection (UTI) antecedent was observed in 31 cases (70.5\%), and of those, 24 cases of UTI were accompanied by fever. All children presented micturition urgency. Daily urinary incontinence was observed in 33 cases (75\%) and nocturnal enuresis in $23(52.3 \%)$. As for micturition frequency, 15 (34.1\%) had normal frequency $19(43.2 \%)$ presented more than 10 daily micturition episodes and $10(22.7 \%)$ thought they urinated less than 5 times a day. In the uroflowmetry and electromyography examination, 14 (31.8\%) experienced lack of coordination during micturition. Of 10 children with infrequent micturition, 5 confirmed this in their micturition diaries and 2 listed more than 5 micturition episodes per day in the diary. Of 19 patients presenting polaciuria, only 5 confirmed this in their micturition diaries, while 7 had less than 10 micturition episodes per day.

Conclusion: Most children with LUTD presented a previous UTI, and daily incontinence was verified in around $75 \%$ of the patients. Complaints of polaciuria or infrequent micturition are not noted completely in the micturition diaries and there is no parameter in the clinical history that offers good sensitivity or specificity for the diagnosis of lack of perineal coordination.
\end{abstract}

Key words: children; urination disorders; urinary tract infections; neurogenic bladder

Int Braz. J Urol. 2006; 32: 70-6

\section{INTRODUCTION}

Lower urinary tract dysfunction (LUTD) is a clinical term related to urination disorders that occur in children without neurological alterations. The dysfunction is related to vesicoureteral reflux (present in 15 to $50 \%$ of patients with vesical instability) $(1,2)$ and urinary infection in children is an important risk factor for renal scars and secondary arterial hypotension (3-6).

The largest incidence occurs in children between 3 and 7 years of age and is featured in girls at a rate of 9:1 compared to boys (7). It significantly affects the patient's quality of life, possibly leading to psychological and behavior alterations (8). In children with nocturnal enuresis and urinary incontinence 
with low self esteem, it has already been demonstrated that there is a normalization of the general psychological picture with a successful treatment of incontinence (9).

It is clinically presented with urination urge and urge incontinence, with the possibility of other symptoms such as polaciuria, infrequent urination and suprapubic or perineal pain.

In relation to urodynamic findings, LUTD can be divided into 2 different groups (10). In the first group we observe intense detrusor non-inhibited contractions during the bladder filling phase that is opposed to pelvic floor muscle contractions and maneuvers to retain the urine, such as the Vincent maneuver (11) and crossing the legs. In the second group, there is incomplete relaxation of the sphincter blocking urination and causing a prolonged or interrupted urinary stream.

The objective of the present study was to prospectively assess the clinical presentation of children with LUTD, correlating with objective data such as urinary diary and uroflowmetry with electromyogra- phy and assessing the sensitivity and the specificity of parameters of the clinical history in relation to the exams utilized. To our knowledge, there are no available data in the literature that use this type of correlation.

\section{MATERIALS AND METHODS}

Forty-four children with LUTD (36 girls and 8 boys, with a mean age of 6.8 years, varying from 3 to 17 years) were retrospectively assessed by means of a specific questionnaire that analyzed the clinical presentation of those patients (Table-1). This questionnaire was applied jointly to parents and children. The initial assessment protocol was composed of a clinical symptoms questionnaire, simple abdomen radiography and a voiding cystourethrogram (in the event of a history of urinary infection), uroflowmetry with electromyography and ultrasonography of the urinary apparatus with a measure of residual urine. The electromyography was performed together with the uroflowmetry, with surface electrodes placed in

Table 1 - Questionnaire aimed at parents. Each question was fully explained to the interviewee so that he/she could understand.

\begin{tabular}{lll}
\hline & No & Yes \\
\hline 1. Urgency & $($ ) & $($ ) \\
2. Urge incontinence & $($ ) & $($ ) \\
3. Loss without urgency & $($ ) & $($ ) \\
4. Polaciuria & $($ ) & $($ ) \\
5. Infrequent urination (up to 3 times a day) & $($ ) & $($ ) \\
6. Giggle incontinence & ( ) & ( ) \\
7. Stress incontinence & ( ) Continuous & ( ) Intermittent \\
8. Urination difficulty & ( ) Present & ( ) Absent \\
9. Nocturia (wake up at night to urinate) & ( ) & ( ) \\
10. Type of urinary stream & ( ) & ( ) Daily \\
11. Vincent maneuver & ( ) More than 10 episodes per month \\
12. Abdominal pain & ( ) Between 3 and 10 episodes per month \\
13. Vaginal discharge & ( ) Less than 3 episodes per month \\
14. If there is diurnal urinary incontinence, what is the frequency & &
\end{tabular}


the perineal and abdominal region to also register the activity of the rectus abdominal during urination. With this assessment, the child's urodynamic is studied in a non-invasive manner allowing us to correctly classify the LUTD. The performance of the complete urodynamic study (invasive) remained restricted to the cases where there was upper urinary tract dilation associated to or present in those children with symptoms that were unreceptive to clinical treatment. The symptomatology of patients was then compared to the findings in both the urination diary and the uroflowmetry, as they are objective parameters.

The criteria of exclusion were neurological alterations or clinical signals suggesting spina bifida occulta, such as tufts of hair, lipomas and spots in sacral region, or anatomic alterations, such as posterior urethral valve, ureterocele and ectopic ureter.

The statistical analysis was performed through an assessment of the frequency symptoms related to the disease and the sensitivity and specificity of the various forms of clinical presentation and the complementary clinical exams used. The sensitivity and specificity were calculated using the following formulas: number of patients with a certain symptom divided by the number of children with sphincteric dysfunction, and number of children without symptoms divided by the number of children without sphincteric dysfunction.

\section{RESULTS}

Previous urinary infection showed up in 31 cases $(70.5 \%)$, with $24(54.5 \%)$ presenting episodes of UTI with fever. The distribution of the clinical presentation frequencies is demonstrated in Table2.

In terms of urinary frequency, $15(34.1 \%)$ had normal frequency, $19(43.2 \%)$ voided more than 10 times a day and $10(22.7 \%)$ patients thought they urinated less than 5 times a day. Correlating clinical symptoms with objective data, we observed that of the 10 children with infrequent urination, 5 confirmed this in their urination diaries. Of the 19 patients claiming polaciuria, only $5(26 \%)$ confirmed this in the diary, while 7 (37\%) urinated less than 10 times per day and 7 did not fill out the diary. These figures are represented in Table-3.

The frequency of maneuvers to retard urination, such as the Vincent maneuver and crossed legs, as well as constipation frequency (characterized as more than 2 days without defecating) and encopresis, are demonstrated in Table-2. The occurrence of occasional suprapubic region pain and vaginal running are also registered in this Table.

Even though cystourethrogram was indicated in all cases with urinary infection [31], only 23 children took it. A presence of vesicoureteral reflux was verified in 3 patients (13\%).

In the uroflowmetry assessment with electromyography, 14 patients $(31.8 \%)$ experienced lack of coordination in micturition (Figure-1). Only 5 patients were submitted to a complete urodynamic study. Sensitivity and specificity of some parameters of the clinical history in relation to the incoordination in micturition obtained by means of uroflowmetry with electromyography were respectively: Vincent maneuver - 50\% and 68\%; crossing the legs $-66 \%$ and $45 \%$; urination difficulty $-41 \%$ and $71 \%$; interrupted stream $-25 \%$ and $63 \%$; and constipation $-30 \%$ and $68 \%$.

Table 2 - Distribution of clinical presentations on low urinary tract dysfunction in 40 children.

\begin{tabular}{ll}
\hline Clinical Presentation & Number of Patients (\%) \\
\hline UTI & $31(70.5)$ \\
UTI with fever & $24(54.5)$ \\
Urgency & $40(100)$ \\
Urge incontinence & $33(75)$ \\
Nocturnal enuresis & $23(53.2)$ \\
Giggle incontinence & $16(36.4)$ \\
Voiding effort & $12(27.3)$ \\
Constipation & $12(27.3)$ \\
Encopresis & $2(4.5)$ \\
Suprapubic pain & $19(43.2)$ \\
Vaginal discharge & 12 from 36 girls (33.3) \\
Maneuver to cross the legs & $18(40.9)$ \\
Vincent Maneuver & $13(29.5)$ \\
\hline
\end{tabular}


Table 3 - Correlation between reported urination frequency observed through clinical history and through the urination diary.

\begin{tabular}{lllccc}
\hline \multicolumn{5}{l}{ Number of Urinations in the Diary } \\
\hline Symptoms (anamnesis) & $<5$ urinations & $5-9$ & $>10$ & Without diary & Total \\
Infrequent urination & 5 & 2 & 0 & 3 & 10 \\
Polaciuria & 0 & 7 & 5 & 7 & 19 \\
\hline
\end{tabular}

\section{COMMENTS}

Even though the LUTD is well described in children, few prospective studies have been accomplished and, to our knowledge, none have assessed the incidence of the most frequent urinary claims. As well, we do not know if the symptoms are well correlated to the objective data obtained from the urination diary, the ultrasonography assessment of the residual urine and the uroflowmetry with electromyography. LUTD in the child is a clinical condition that predominantly affects girls. Hanna et al. have noted an incidence of $90 \%$ in girls (7). In the present study, the larger prevalence in females is confirmed $(81.8 \%)$. The most affected age group was from 3 to 7 years of age (7). In this study, the mean age was 6.8 years.

The relationship between LUTD and urinary infection is of utmost importance in the follow-up of those children, and it presents therapeutic and prognostic implications. Children with vesicoureteral reflux (VUR) and LUTD have a larger chance of refractory infection than those children without LUTD. Snodgrass found that children with VUR and LUTD presented refractory infection in $44 \%$ of cases (12), while those with only VUR had an incidence of $11 \%$. A precedent urinary infection was observed in $70.5 \%$ of children assessed in this study; of those, $77.4 \%$ had already presented some episode of UTI with fever.

Among the symptoms associated with LUTD, urgency and urge incontinence are the most prevalent. Urgency was observed in all child studies, with reports of diurnal urinary incontinence found in $75 \%$ of the cases. Nocturnal enuresis (52.3\%) and urina- tion difficult (27.3\%) were also frequent complaints. Giggle incontinence was a symptom present in $23 \%$ of children with diurnal urination symptoms (13), having been observed in $36.4 \%$ of children accessed in the present study. In an analysis of 1421 children 5 to 15 years of age, Chandra et al. noticed the presence of laugh urinary incontinence in 109 (7.7\%) (13). Alterations in urinary frequency were found in $66 \%$ of children. Among assessed patients, $43.2 \%$ reported that void more than 10 times a day, while $22.7 \%$ thought that they urinated less than 5 times a day.

In the attempt to retain urine in the bladder, the child tends to contract the pelvic muscles, sometimes assuming the classic posture to achieve that (Vincent maneuver) (11), and crossing the legs. Maneuvers to retain the urine in the bladder were observed in at least half the children studied. Children with detrusor instability that use postural maneuvers to retain urine have a higher incidence of urinary tract infection than those who do not try to obstruct the urinary flow (14).

It is already known that alterations in intestinal habits have a great influence on the function of the low urinary tract and can be associated with a syndrome that Koff et al. described as elimination dysfunction (15). Among children studied, 27.3\% claim constipation and $4.5 \%$ presented encopresis. The association of LUTD in girls with some degree of vaginal discharge was not found in the literature, but it was observed in the current study in one third of the female cases. The hypothesis is that there is a urine reflux in the vagina of the girls with LUTD, which generates colpitis and vaginal discharge; however, there is a need for more studies in this area in order to prove this correlation. 

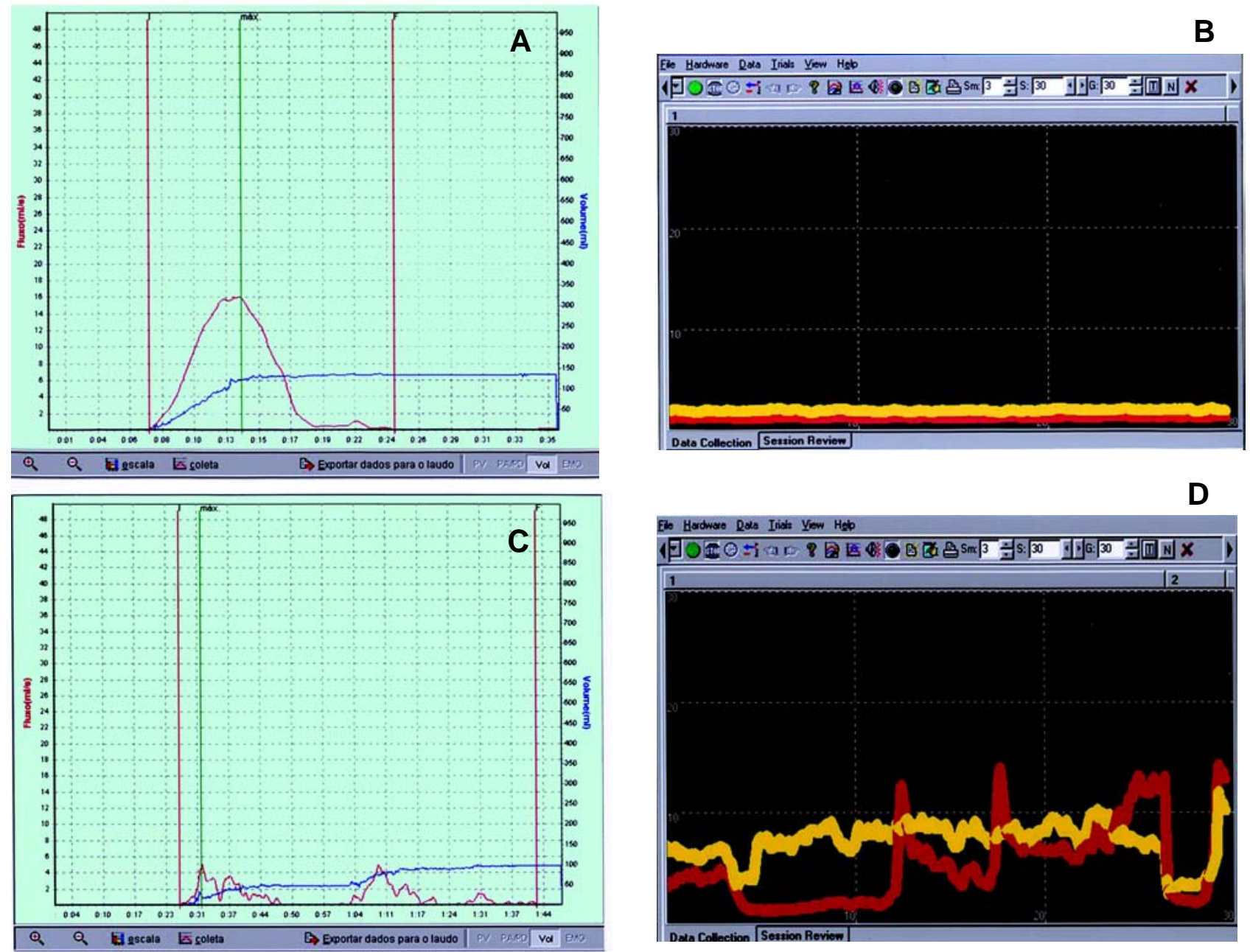

Figure 1 - Normal child, presenting uroflowmetry with normal urinary flow spinning top shape (A) and electromyography with absence of perineal and abdominal muscular activity $(B)$. Child with interrupted urinary flow $(C)$ and sphincteric incoordination, with electromyography evidencing abdominal and perineal activity during urination (D).

The association between urinary tract infection, vesicoureteral reflux, LUTD and the formation of renal scars is already well established. Data in the literature show the presence of VUR in 15 to $50 \%$ of patients with vesical instability $(1,2)$. In children with LUTD assessed per voiding cystourethrogram (VCU) in this study, the occurrence of RVU was observed in $13 \%$ of the cases. Due to the improvement in reflux with the clinical treatment of LUTD and the low incidence of RVU in this group of patients $(1,16)$, we should discuss the need to perform a VCU in these cases. In cases of LUTD, a cystourethrogram can not only assesses the presence of associated vesicoureteral reflux, but also demonstrates the signals of vesical instability (spinning top urethra, serrulated bladder wall, bladder elongation and, in extreme cases, presence of diverticula). In analyzing 193 children with RVU, Barroso et al. found sings of vesical instability in the cystourethrogram in $26 \%$ of the patients (17). These findings demonstrated that among children that presented symptoms of LUTD, $64 \%$ had cystourethrogram. However, no study that came to our knowl- 
edge assessed the prognostic value of these findings in this group of patients.

It is of utmost importance for the treatment of children with LUTD that a differential diagnosis be performed regarding the urge syndrome, urination dysfunction (sphincteric dysfunction) and the lazy bladder syndrome. In order to make this differential diagnosis, data alone from the patient's clinical history are imprecise and insufficient (18). The weak correlation between the symptomatology presented by the patient and the objective results of complementary exams was confirmed in this study. Of the 10 children reporting infrequent urination, half confirmed this finding through their urination diaries. Of the 19 patients with polaciuria, only $26.3 \%$ confirmed this in the diary. Sensitivities and specificities of parameters from the clinical history in relation to the objectives findings of voiding incoordination obtained through uroflowmetry with electromyography were equally low. The discovery of maneuvers to retain urine presented little correlation with the diagnosis of lack of perineal coordination. As well as the report of urination difficulty and the claim of interrupted stream, Vincent maneuvers (11) and crossing the legs showed a weak correlation with the results obtained through the non-invasive urodynamic assessment. Due to the low correlation between the symptoms of LUTD and the results of the exams, the present study shows that there is little correlation between LUTD symptoms and the assessment through the urination diary and uroflowmetry with electromyography. This suggests a need to perform a noninvasive urodynamic to classify children with lower urinary tract dysfunction.

\section{CONFLICT OF INTERESTS}

None declared.

\section{REFERENCES}

1. Koff SA, Lapides J, Piazza DH: Association of urinary tract infection and reflux with uninhibited bladder contractions and voluntary sphincteric obstruction. J Urol. 1979; 122: 373-6.
2. Snodgrass W: Relationship of voiding dysfunction to urinary tract infection and vesicoureteral reflux in children. Urology. 1991; 38: 341-4.

3. Varlam DE, Dippell J: Non-neurogenic bladder and chronic renal insufficiency in childhood. Pediatr Nephrol. 1995; 9: 1-5.

4. Naseer SR, Steinhardt GF: New renal scars in children with urinary tract infections, vesicoureteral reflux and voiding dysfunction: a prospective evaluation. J Urol. 1997; 158: 566-8.

5. Barroso U Jr, Barroso DV, Jacobino M, Vinhaes AF, Macedo A Jr, Srougi M: Etiology of urinary tract infection in scholar children. Int Braz J Urol. 2003; 29: 450-4.

6. Barroso U Jr, Jednak R, Barthold JS, Gonzalez R: Outcome of ureteral reimplantation in children with the urge syndrome. J Urol. 2001; 166: 1031-5.

7. Hanna MK, Di Scipio W, Suh KK, Kogan SJ, Levitt SB, Donner K: Urodynamics in children. Part II. The pseudoneurogenic bladder. J Urol. 1981; 125: 534-7.

8. von Gontard A, Lettgen B, Olbing H, Heiken-Lowenau C, Gaebel E, Schmitz I: Behavioural problems in children with urge incontinence and voiding postponement: a comparison of a paediatric and child psychiatric sample.. Br J Urol. 1998; 81 (3 Suppl): 100-6

9. Hagglof B, Andren O, Bergstrom E, Marklund L, Wendelius M. Self esteem before and after treatment in children with nocturnal enuresis and urinary incontinence. Scand J Urol Nephrol. 1997;158: 1035-9.

10. van Gool JD: Dysfunctional voiding: a complex of bladder/sphincter dysfunction, urinary tract infections and vesicoureteral reflux. Acta Urol Belg. 1995; 63: 27-33.

11. Vincent SA: Postural control of urinary incontinence. The curtsy sign. Lancet. 1966; 2: 631-2.

12. Snodgrass $\mathrm{W}$ : The impact of treated dysfunctional voiding on the nonsurgical management of vesicoureteral reflux. J Urol. 1998; 160: 1823-5.

13. Chandra M, Saharia R, Shi Q, Hill V: Giggle incontinence in children: a manifestation of detrusor instability. J Urol. 2002; 168: 2184-7; discussion 2187.

14. Hellerstein S, Linebarger JS: Voiding dysfunction in pediatric patients. Clin Pediatr (Phila). 2003; 42: 43-9.

15. Koff SA, Wagner TT, Jayanthi VR: The relationship among dysfunctional elimination syndromes, primary vesicoureteral reflux and urinary tract infections in children. J Urol. 1998; 160: 1019-22.

16. Bratslavsky G, Feustel PJ, Aslan AR, Kogan BA: Recurrence risk in infants with urinary tract infections and a negative radiographic evaluation. J Urol. 2004; 172: 1610-3; discussion 1613. 
17. Barroso U Jr, Vinhaes AJ, Barros M, Barroso VA, Calado AA, Zerati Filho $M$. Findings in cystourethrography that suggest lower urinary tract dysfunction in children with vesicoureteral reflux. Int Braz J Urol. 2004; 30: 504-7.
18. Barroso U Jr, Macedo A Jr: Disfunção do trato Urinário inferior em crianças. In: Macedo A Jr, Lima SVC, Streit D, Barroso U Jr. Urologia Pediátrica, Roca. 2004; pp. 33-52.

Accepted after revision: August 31, 2005

Correspondence address:

Dr. Ubirajara Barroso Jr.

Rua Alameda dos Antúrios, 212 / 602

Salvador, BA, 40280-620, Brazil

E-mail: ubarroso@uol.com.br 